\title{
Numerical Investigation into the Effects of Tip Clearance on the Performance of a Counter-Rotating Axial Flow Compressor
}

\author{
T. Liang ${ }^{1}$, B. Liu ${ }^{1 \dagger}$, S. Spence ${ }^{2}$, X. Mao ${ }^{1}$ and H. Cheng ${ }^{1}$ \\ ${ }^{1}$ School of Power and Energy, Northwestern Polytechnical University, Xi'an, Shaanxi, 710072, P. R. China \\ ${ }^{2}$ School of Mechanical and Aerospace Engineering, Queen's University Belfast, Belfast, BT9 5AH, UK
}

†Corresponding Author Email: liubo704@nwpu.edu.cn

(Received November 21, 2019; accepted February 3, 2020)

\begin{abstract}
The impact of varying the tip clearance of each rotor on the performance of a counter-rotating axial compressor has been investigated based on numerical simulations. The main purpose was to investigate the sensitivity to the tip clearance of each of the two individual rotors and the corresponding aerodynamic mechanisms associated with the performance variation in this compressor. The results indicated that both the total pressure ratio and the efficiency decreased as the tip clearance was increased, and the sensitivity curve for peak efficiency for both rotors was found to be an approximately linear negative relationship with increasing tip clearance. The variations of peak efficiency and stability margin of Rotor 2 were more sensitive to changing tip clearance than Rotor 1 . An optimum combination of tip gaps existed for this compressor, i.e. $0.5 \tau$ for Rotor 1 and $0.25 \tau$ for Rotor 2 (where $\tau$ represents the nominal tip clearance value). At this optimum configuration, the peak efficiency and stability margin were improved by $0.63 \%$ and $29.4 \%$, respectively. The location of the onset of the tip leakage vortex was found to be shifted downstream when the tip clearance increased. The nature of the tip leakage flow for each rotor was found to be influenced by the variation of tip clearance in the other rotor. Rotor 2 showed a more significant impact on Rotor 1. Additionally, varying the combination of tip clearances changed which of the two rotors was the first to stall.
\end{abstract}

Keywords: Counter-rotating compressor; Tip clearance; Efficiency; Tip leakage flow; Absolute vorticity.

\section{NOMENCLATURE}

$\begin{array}{ll}\text { Cp } & \text { static pressure coefficient } \\ \text { CRAC } & \text { Counter-Rotating Axial Flow Compressor } \\ \text { IGV } & \text { Inlet Guide Vane } \\ \text { LE } & \text { Leading Edge } \\ \text { NS } & \text { Near Stall } \\ \text { OGV } & \text { Outlet Guide Vane } \\ \text { R1 } & \text { clockwise rotating rotor } \\ \text { R2 } & \text { anti-clockwise rotating rotor } \\ \text { SM } & \text { Stability Margin } \\ \text { TE } & \text { Trailing Edge } \\ \text { TLA } & \text { Tip Leakage Flow Angle }\end{array}$

\section{INTRODUCTION}

It is well known that the existence of tip clearance in a compressor is inevitable and it should actually exist as a definite space between the moving parts and stationary casing wall avoiding the physical contact with the casing, possibility of exciting blade flutter, accommodating radial growth due to centrifugal stresses and thermal expansion, also

\author{
TLF Tip Leakage Flow \\ TLV Tip Leakage Vortex \\ TLVR Tip Leakage Velocity Ratio \\ $y+\quad$ non-dimensional wall distance \\ $\eta \quad$ adiabatic efficiency \\ $\pi \quad$ total pressure ratio \\ $\tau \quad$ nominal tip clearance \\ $\Psi \quad$ non-dimensional mass flow rate
}

accommodating rotor displacement due to critical speeds, and to a lesser extent manufacturing variations. Nevertheless, it has been studied from a long time ago due to its immoderate loss on the compressor performance. And in particular, tip leakage flow (TLF) across the rotor tip gap has a profound effect on the performance and stability of a compressor. As much as $30 \%$ of the total loss in an axial compressor has been attributed to the flow 
through the tip clearance (Storer \& Cumpsty 1994), which has been a principal research topic for turbomachinery designers for decades (Vo et al. 2008; Du et al. 2013; Tan et al. 2015; Boudet et al. 2015). The size of the tip clearance is very significant, and much effort has been expended to gain a better understanding of the impact of tip clearance size on compressor performance and stability (Lejon et al. 2015; Yamada et al. 2013; Berdanier \& Key 2016). The results presented by Sakulkaew et al. (2013) indicated that for clearances gap $(0.8 \%-3.4 \%$ span $)$, isentropic efficiency is deteriorated with an increase in clearance. It is less sensitive to tip clearance for gaps greater than $3.4 \%$. Domercq \& Escuret (2007) deduced that the tip clearance flow and its gap size variation have a major impact on the performance and stability of high-pressure compressors.

Past research has indicated that both TLF and increasing the tip clearance size play very important roles in the compressor performance, as measured in terms of stability margin, pressure rise, and efficiency. Recently, the counter-rotating axial flow compressor (CRAC) has attracted greater attention because the configuration of two counter-rotating rotors allows the stator blade row between the two adjacent rotors to be eliminated (Schimming 2003; Joly et al. 2013), which offers the potential for the overall compressor to become axially shorter and much lighter. To gain further insights into the flow physics in a CRAC, much research work has been conducted on the effect of important factors, including axial spacing between the two rotors, inlet distortion, and unsteady effects, etc. (Sharma et al. 1998; Nouri et al. 2013; Mistry \& Pradeep 2014; Shi et al. 2015). That work has revealed many new characteristics and flow phenomena that are unique to the CRAC by comparison with a conventional axial compressor stage. For example, one of the counter-rotating stages might produce a stall-free characteristic for certain rotor under some conditions (Sharma et al. 1988; Pundhir \& Sharma 2013).

Compared with a conventional stage, another unique flow phenomenon that exists in the CRAC is that the TLF from the upstream rotor directly affects the flow in the tip region of the downstream rotor instead of a stator. Additionally, an increase of tip clearance size would intensify the impact of this rotor-rotor interaction on the performance of the CRAC. However, investigations of the detailed effects of changing tip clearance size in CRAC are limited in the open literature. Thus, the motivation of the present work is to contribute to a better understanding of the impact of varying tip clearance of each individual rotor on the performance of a CRAC while taking account of the rotor-rotor interaction.

The paper is organized as follows: after describing the CRAC used in this investigation, the numerical method and its validation are shown in the upcoming section. Thereafter, the numerical results and corresponding detailed discussions are presented in a later section. Finally, concise conclusions are shown in the last section.

\section{EXPerimental COMPRESSOR TeSt Rig AND NUMERICAL METHOD}

\subsection{Description of the Compressor Test Rig}

The compressor test rig studied in this paper is a low-speed counter-rotating axial flow compressor at the National Defense Aerodynamics Laboratory of Airfoil and Cascade in Northwestern Polytechnical University in China. Three pictures of the CRAC are shown in Fig. 1. The CRAC included four blade rows, i.e. inlet guide vane (IGV) with 22 blades, a clockwise rotating rotor (Rotor 1), an anticlockwise rotating rotor (Rotor 2), and an outlet guide vane $(\mathrm{OGV})$ with 32 blades. The two rotors were driven through gearboxes by two AC electric motors with frequency controllers, which allowed the two rotors to run at any speed combination from 0 to 8000 RPM. The total pressure ratio and mass flow of the CRAC at the design condition was about 1.22 and $6.4 \mathrm{~kg} / \mathrm{s}$ respectively. Table 1 presents some of the other principal parameters of the compressor

Table 1 Principal parameters of the two rotors

\begin{tabular}{|c|c|c|}
\hline Design Parameters & Rotor 1 & Rotor 2 \\
\hline Tip clearance(mm) & 0.5 & 0.5 \\
\hline Blade number & 19 & 20 \\
\hline Tip speed(m/s) & 167.6 & 167.6 \\
\hline Hub-tip ratio & 0.485 & 0.641 \\
\hline Rotational speed(rpm) & 8000 & 8000 \\
\hline Blade tip diameter(m) & 0.2021 & 0.2026 \\
\hline Tip blade chord(m) & 0.0832 & 0.0769 \\
\hline
\end{tabular}

\subsection{Numerical Methodology and Validation}

The numerical simulations in this study were performed using a commercial solver package, Fine/Turbo from NUMECA International. The steady, compressible, three-dimensional Reynolds Averaged Navier-Stokes equations were solved using the Spalart-Allmaras turbulence model in a relative coordinate frame. The equations were discretized in space using a cell-centered finite volume scheme developed by Jameson, and the discretization in time was done using the explicit fourth-order Runge-Kutta scheme in the steady simulations. Total pressure $(101325 \mathrm{~Pa})$ and total temperature $(288.15 \mathrm{~K})$ were applied at the inlet boundary. Non-slip and adiabatic boundary conditions were given on the solid walls. An average static pressure boundary condition was provided on the outlet plane in the steady computations. The matching mixing plane methods were conducted on the rotor interface, which has been proved by Deng et al. (2013) to be in a better agreement with the experimental data compared with other rotor interface methods in CRAC. Fig. 2(a) presents the computational domain and boundary conditions. Additionally, Multi-grid techniques and residual smoothing methods were 
adopted to speed up residual convergence in the computations.

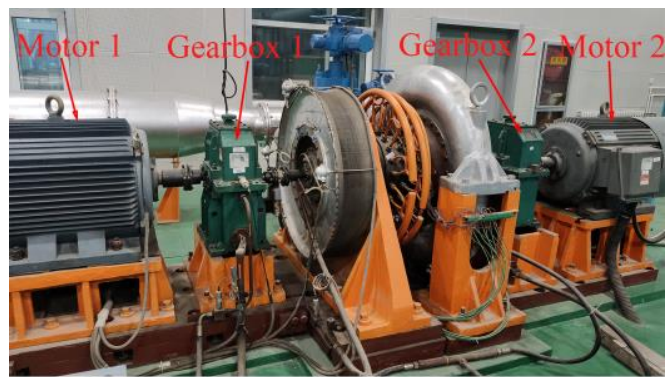

(a) Global View

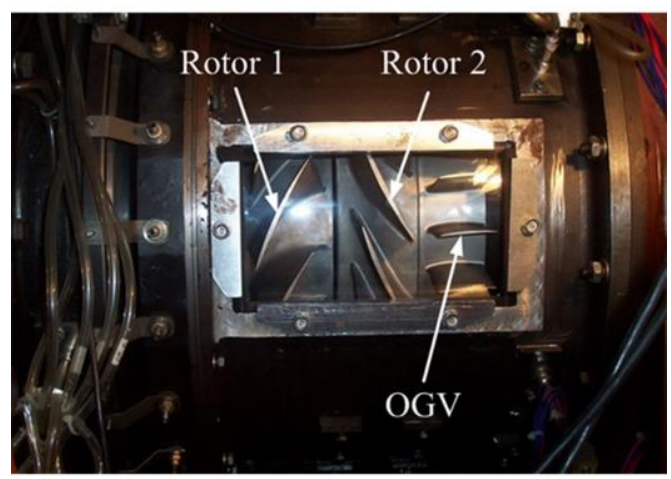

(b)Enlarged view at blades

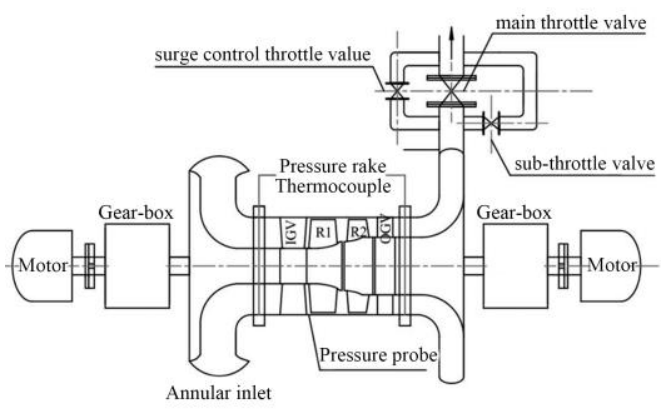

(c)Structure of experiment platform

Fig. 1. Two-stage counter-rotating axial flow compressor.

The computational mesh of the main flow passage was generated with $\mathrm{O} 4 \mathrm{H}$ topology using the AUTOGRID5 commercial software. Butterfly topology was chosen to model the real tip gap in both rotors. The grid independence was verified by Wang et al. (2015) in a study at the design point for the CRAC. The results showed that the overall performance showed no significant variation for mesh sizes exceeding about $2.03 \times 10^{6}$ elements. Therefore, considering both simulation accuracy and computing efficiency, a total grid size of about $2.4 \times 10^{6}$ elements was used for simulating one passage (comprising four blade rows). A minimum grid spacing of $4 \times 10^{-6} \mathrm{~m}$ was applied at the solid walls, which resulted in a $y+$ value no more than 5 at the walls. Fig. 2(b) shows the computational mesh of the two rotors, together with enlarged views of the grid near the tip leading edge (LE) and trailing edge (TE).

In order to validate the numerical methodology used in the current paper, a series of steady simulations were carried out to obtain the performance maps of the CRAC at the design speed of $8000 \mathrm{rev} / \mathrm{min}$ using the nominal tip clearance. The last convergent

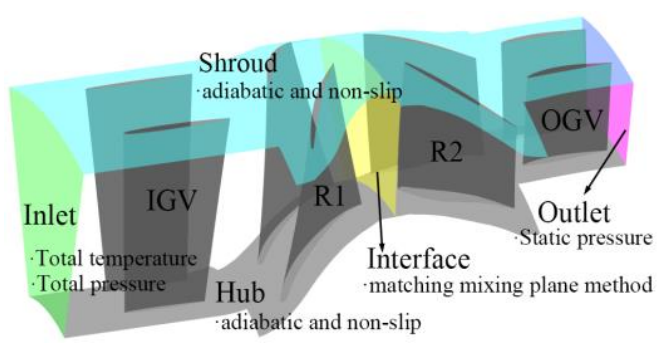

a) computational domain

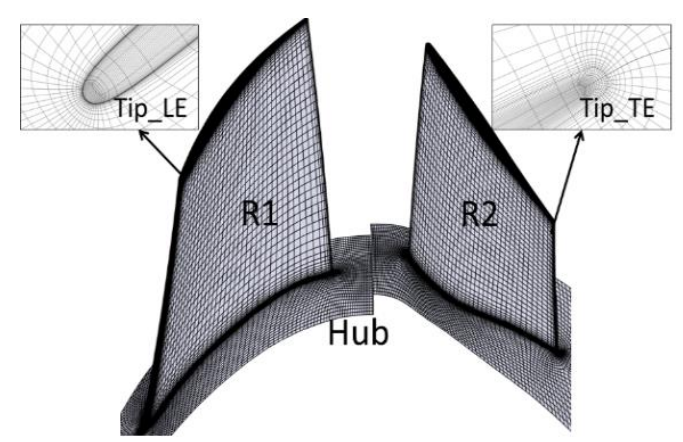

b) computational mesh of the two rotors

Fig. 2. Computational domain and mesh of the two rotors.

point was used as the criterion for determining the stall flow rate of the compressor. In order to calculate the stability margin of the CRAC as accurately as possible, the back-pressure dichotomy method was used in the numerical simulation (Gao et al. 2012). The outlet static pressure and its step variation were both updated gradually as the operating point approached the numerical stall condition. The experimental and simulated performance characteristics are plotted in Fig. 3 as a function of mass flow coefficient normalized by the choking mass flow rate. The reason for the scattering in the experimental data is that the experimental data presented contain several groups of data collected under different environmental conditions, which can represent the actual characteristics of the compressor more reliably. One can see that the overall performance characteristics agree very well over most operating range. The close comparison of the performance curves gives confidence in the numerical methodology used.

The effects of varying the tip clearance of each rotor on the performance of the CRAC were investigated using the computational model. Specific schemes are as follows: when the clearance of rotor 2 is maintained at the design gap of $1.0 \tau$, rotor 1 corresponds to 11 different tip clearances, which are respectively $0.1 \tau, 0.25 \tau, 0.5 \tau, 0.75 \tau, 1 \tau$, 
$1.25 \tau, 1.5 \tau, 1.75 \tau, 2 \tau, 2.25 \tau, 2.5 \tau$; When the clearance of rotor 1 is maintained at design gap of $1.0 \tau$, rotor 2 corresponds to 11 different tip clearances, which are $0.1 \tau, 0.25 \tau, 0.5 \tau, 0.75 \tau, 1 \tau$, $1.25 \tau, 1.5 \tau, 1.75 \tau, 2 \tau, 2.25 \tau$ and $2.5 \tau$, respectively. It should be mentioned that the IGV and OGV blades were designed with a relatively large axial distance from R1 and R2. Therefore, the following analyses are mainly performed for R1 and R2 due to the effect of the tip clearance changes of two rotors on the flow field in IGV and OGV is insignificant.

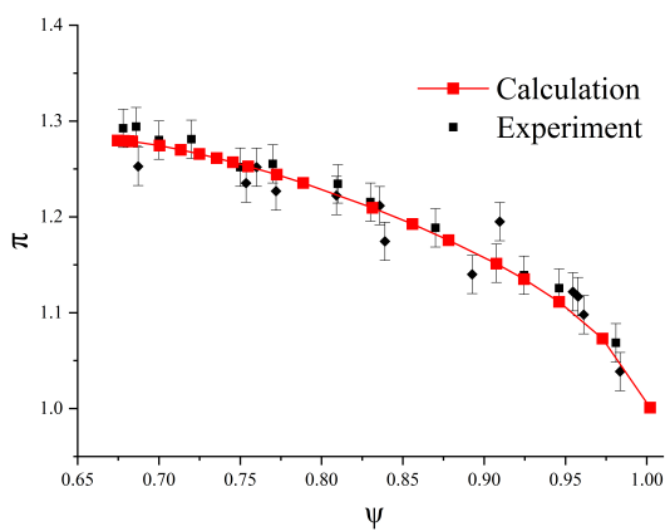

a) Total pressure ratio

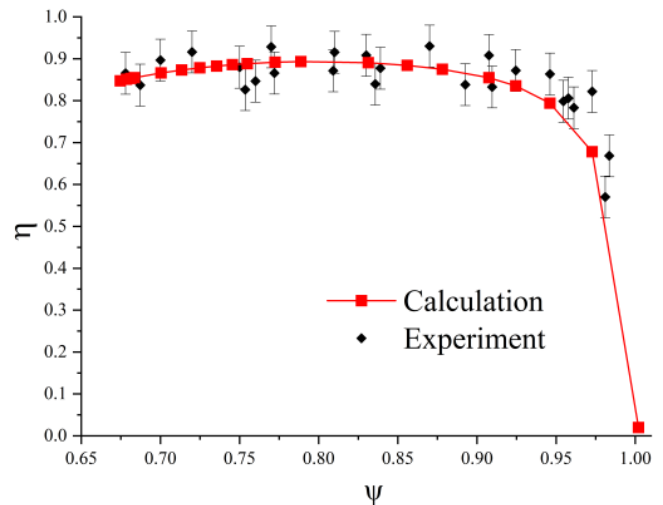

b) Adiabatic efficiency

Fig. 3. Comparison of experimental and calculated overall performance.

\section{RESUlTS AND DisCUSSION}

\subsection{Effects of Tip Clearance on the Overall Performance}

This section describes a parametric study that was carried out to assess the effect of tip clearance on the overall performance. Fig. 4 shows the simulated efficiency and total pressure characteristics for varying tip clearances for Rotor 1 while the clearance for Rotor 2 was maintained constant at the nominal value $(1.0 \tau)$. Fig. 5 shows the corresponding plot for varying clearances in Rotor 2 while Rotor 1 was constant.

It can be seen that when the tip clearance for a particular rotor decreased, the total pressure ratio and efficiency for that rotor increased, and the level of increase was greater for operating conditions near the stall point. Moreover, when the tip clearance of rotor 2 changed, the corresponding change of efficiency and pressure ratio was more significant than for rotor 1. As can be seen in Figs. 4 and 5, the stable working range also reduced with increasing tip clearance and the reduction in stability was greater for clearance increases in rotor 2.

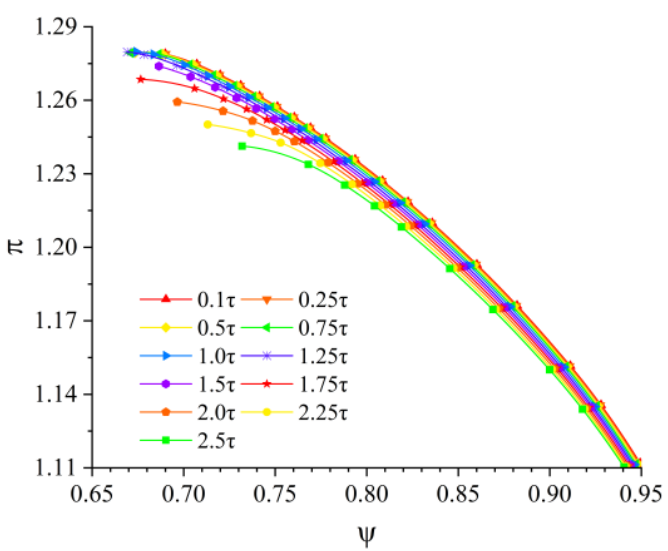

a) Total pressure ratio

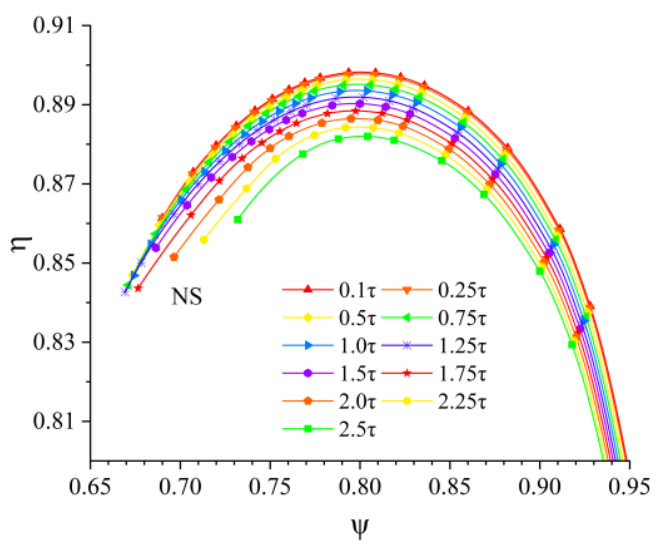

b) Adiabatic efficiency

Fig. 4. Performance for different tip clearances of rotor 1 .

In order to more clearly consider the influence of changing the tip clearance of either rotor on the overall performance of the CRAC, the changes in surge margin $(\triangle S M)$ and peak efficiency $(\Delta \eta)$ due to altering tip clearance in each rotor are presented in Fig. 6. The change in stability margin for the overall CRAC machine was defined using the equation below:

$\Delta S M=\left[\frac{\pi_{\text {stall }} m_{\text {design }}}{\pi_{\text {design }} m_{\text {stall }}}-1\right]_{\mathrm{i}}-\left[\frac{\pi_{\text {stall }} m_{\text {design }}}{\pi_{\text {design }} m_{\text {stall }}}-1\right]_{\tau}$

Where $m_{\text {stall }}$ represents the mass flow rates at last numerical converged point, the subscript i represents various tip clearance size working conditions, and the subscript $\tau$ represents the tip clearance size is the nominal value $(1.0 \tau)$. 


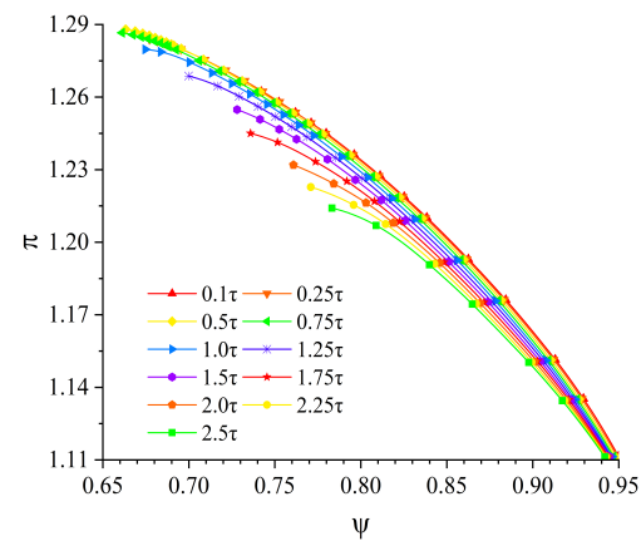

a) Total pressure ratio

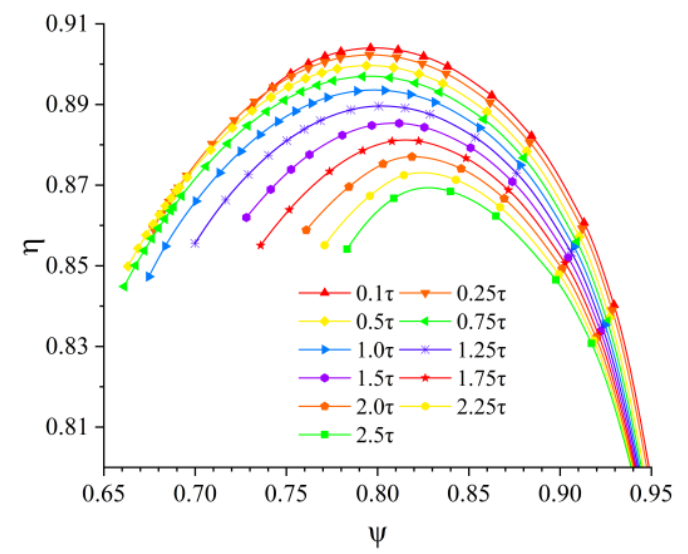

b) Adiabatic efficiency

Fig. 5. Performance for different tip clearances of rotor 2.

It can be seen from Fig. 6 (a) that the peak efficiency sensitivity curve of the two rows of rotors had an approximately negative linear relationship with the tip clearance, and the slope of the sensitivity curve corresponding to Rotor 2 is steeper than that of the Rotor 1. For example, when the tip clearance increased from $0.1 \tau$ to $2.5 \tau$, the peak efficiency of Rotor 1 decreased by only $1.6 \%$, while the peak efficiency of Rotor 2 decreased by $3.5 \%$ for the same change in tip clearance.

Figure 6 (b) shows that the stability margin sensitivity curves of the two rotors had distinctly different trends. The surge margin of Rotor 2 was more sensitive to tip clearance, and the optimum clearance was significantly larger than for Rotor 1 . For Rotor 1, a relatively large tip clearance of $1.75 \tau$ produced the maximum surge margin, with an improvement of $8.8 \%$ over the baseline case of $1.0 \tau$. For most of the range of tip clearance sizes, the stability margin of Rotor 1 was quite insensitive to change in tip clearance. The stability margin of Rotor 2 was best at a relatively small tip clearance of $0.25 \tau$, and it was much more sensitive to changes in tip clearance than Rotor 1 . which is about $28.3 \%$ higher than that under the designed clearance $1.0 \tau$, Considering the effect of tip clearance on the peak efficiency of compressor, there is an optimal clearance combination for rotary compressor, that is, when the tip clearance of rotor 1 is $0.5 \tau$, the tip clearance of rotor 2 is $0.25 \tau$. At this time, the peak efficiency and stability margin of the compressor was increased by about $0.63 \%$ and $29.4 \%$, respectively.

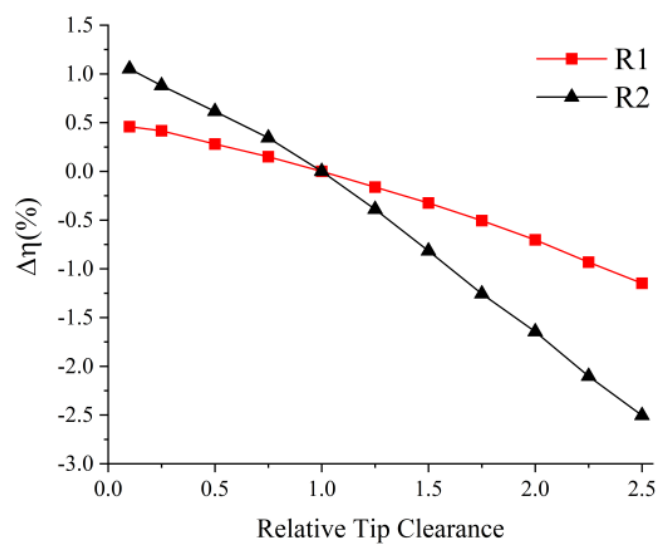

a) Peak efficiency change

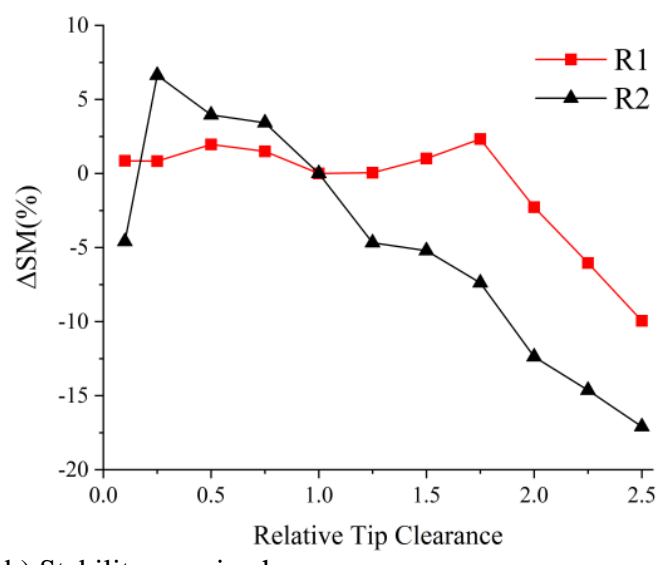

b) Stability margin change

Fig. 6. Changes in compressor stability margin and peak efficiency as a function of tip clearance size.

As a result of the removal of the stator blades between two rows of rotors. The CRAC configuration makes Rotor 2 not only play a pressurizing role, it is also inevitable to convert the work done by Rotor 1 into pressure potential energy, which results in a greater aerodynamic load in Rotor 2, so the tip leakage intensity in Rotor 2 is greater, which will adversely affect the efficiency and stable working range of the compressor. In addition, the flow field through Rotor 1 enters directly into Rotor 2 without any rectifying effect from an intermediate row of stator blades to moderate the non-uniformity and unsteadiness of the flow field. Therefore, the non-uniformity of the flow field from Rotor 1 amplified the nonuniformity of the flow field in Rotor 2, such as the thickening of the inlet boundary layer. Brandt et al. (2002) measured the performance of an isolated compressor rotor with different inlet flow conditions. They found that the thickening of the inlet boundary layer moved the formation of the Tip 


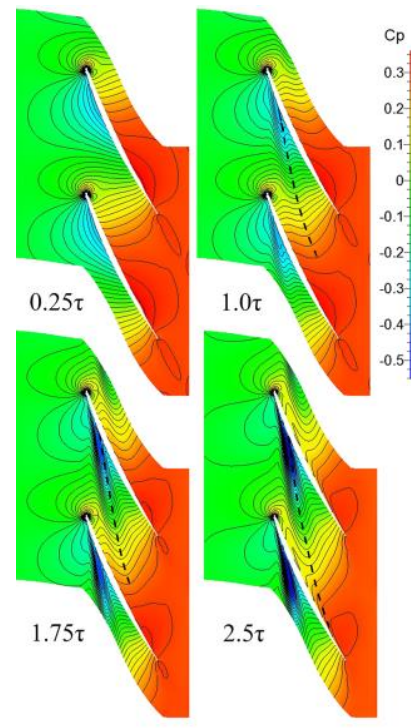

a) R1

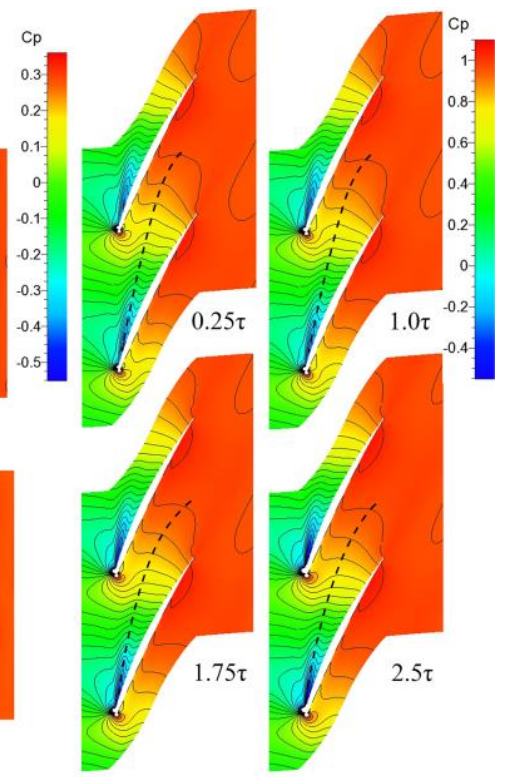

b) R2

Fig. 7. Static pressure coefficient contours of the blade tip near the peak efficiency point with different tip clearance sizes in Rotor 1.

Leakage Vortex (TLV) forward and narrowed the stable operating range of the compressor, which was analogous to increasing the tip clearance size. Since the aerodynamic load on Rotor 2 was larger and the inlet conditions were less uniform, decreasing the tip clearance reduced the intensity of the TLF, and then greatly reduce the mixing and blockage due to the TLF in the second row of blades. Consequently, the peak efficiency and stability margin of the overall CRAC were significantly improved.

\subsection{Effect of Rotor 1 Tip Clearance Change on CRAC}

Figure 7 shows the static pressure coefficient contours of the blade tip near the peak efficiency point with varying tip clearances for Rotor 1 . Inoue \& Kuroumaru (1989) found that the TLV trajectory corresponded to the static pressure chute on the casing. Therefore, the static pressure isoline chute line on the tip section was used to approximately represent the trajectory of the tip leakage vortex, as shown by the black dashed line in Fig.7. From Fig. $7(\mathrm{a})$, there was no obvious leakage vortex trajectory formed at the tip for $0.25 \tau$ clearance, but the static pressure chute of the tip leakage vortex trajectory of Rotor 1 became steeper with increasing tip clearance. The results showed that the size and strength of the TLV increased with the increase of tip clearance.

When the tip clearance increased to $2.5 \tau$, the extent of low energy fluid at the starting position of the TLV in rotor 1 was significantly higher than for the $1.0 \tau$ case. This observation was consistent with the experimental and numerical results of Inoue \& Kuroumaru (1989) and Sakulkaew et al. (2013). They found that increasing tip clearance caused the
TLV trajectory to deviate toward the circumferential direction to the blade leading edge; i.e. the circumferential extent of the TLV increased. When the tip clearance was $2.5 \tau$, the TLV trajectory in rotor 1 intersected the pressure surface of the adjacent blade near the $70 \%$ chord position, resulting in more loss caused by the secondary leakage. Figure 7(b) shows that the variation of the tip clearance of Rotor 1 had little impact on the initial position and the circumferential extent of the TLV in Rotor 2.

In order to further analyze the cause of the change of the leakage flow trajectory in the rotor 2 is not obvious with the increase of the tip clearance size of the rotor 1. Fig. 8 shows the distribution of the tip leakage velocity ratio (TLVR) and tip leakage flow angle (TLA) along the chord length at the middle span of the Rotor 2 tip clearance as the Rotor 1 tip clearance varies at the peak efficiency point. The TLA is defined as the angle of the airflow velocity at the middle position of the tip clearance measured from the tangent to the blade camber line at that point. A larger value of TLA indicates a more intense TLF. Since the TLF is driven by the pressure difference between the suction and pressure sides, the strength of the TLF depends primarily on the blade loading at the tip. The TLVR in the tip clearance is defined as:

$$
T L V R=V_{t l v} / V_{t i p}
$$

Where $V_{t l v}$ represents the leakage velocity in the middle of the tip clearance and $V_{\text {tip }}$ represents the blade velocity at the tip.

It can be seen in Fig. 8 that at the peak efficiency 
point the region of high leakage velocity in Rotor 1 was near the LE of the blade. The distribution of the tip leakage velocity is consistent with the level of loading at the tip of the blade, which was higher near the LE for this blade. So, the loading near the LE is larger, which corresponds to the fact that the TLV in rotor 2 in Fig.7 (b) begins at the LE position under different rotor 1 tip clearance sizes. The leakage velocity near the LE of rotor 2 increases significantly with the increase of the tip clearance of the rotor 1, which indicates that the leakage intensity near the LE of the blade increases. But the change in position of the TLVR corresponding to the maximum value is not significant, which is consistent with the change in the starting position of the leakage flow in Fig.7 (b).

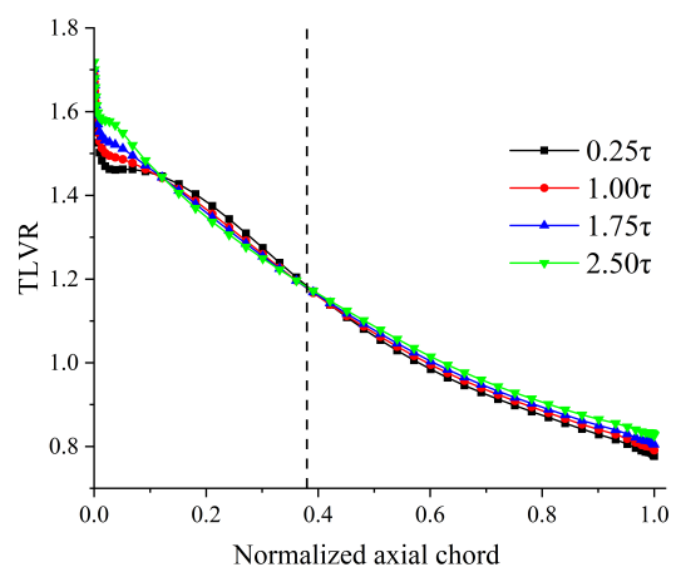

(a)TLVR

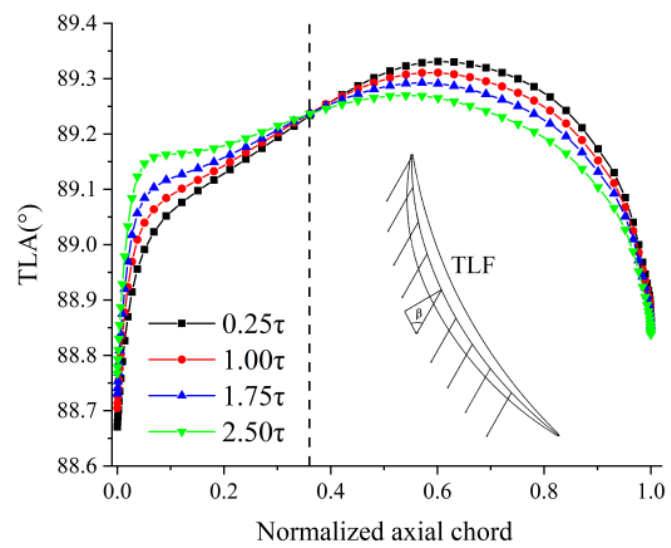

(b)TLA

Fig. 8. TLVR and TLA distribution along with axial chord at the middle of the tip clearance of rotor 2 at peak efficiency condition.

As can also be seen from Fig.8 (b), the amplitude position of the TLA near the LE of the blade corresponds to the position of the maximum load of the blade. The amplitude of rotor 2 TLA near the LE becomes significantly larger with the increase of the tip clearance of rotor 1 . But the amplitude position is almost unchanged. Although the corresponding TLA decreases to a certain extent after $38 \%$ of the axial chord length position of the blade, it can be seen from Fig. 8 (a) that the leakage velocity in the middle and rear of the blade increases. The magnitude of the leakage intensity is the result of the joint action of the leakage velocity and the leakage flow angle, so there is no obvious change in the leakage flow trajectory in Fig.7 (b). Therefore, it can be concluded that at the peak efficiency point, with the increase of rotor 1 tip clearance, the scale and intensity of TLF in rotor 1 channel become larger, and the leakage trajectory gradually deflects along the circumferential direction to the blade frontal line. The influence on rotor 2 is mainly concentrated at the area near LE of the blade, the TLVR and TLA increase, but the initial position of leakage flow and the expansion range in the blade channel remain basically unchanged.

The existing research results indicated that CRAC might produce a stall-free characteristic for certain rotor under some conditions (Pundhir \& Sharma 2013; Sharma et al. 1988), and the larger tip clearance size will have a significant effect on the initial position and leakage intensity of TLV. And the results of Camp \& Day (1997) show that if the instability of the compressor occurs when the pressure ratio characteristic increases, that is, the slope is negative, the initial disturbance type of the compressor at the near stall point is the spike-type.

According to the characteristic curves of the total pressure ratio in Fig. 4 and 5, it can be judged that the CRAC under each tip clearance studied in this paper is a spike-type. Vo et al. (2008) proposed two necessary conditions for judging the occurrence of the spike-type stall, namely, LE spillage and trailing edge backflow, but the corresponding flow rates of these two phenomena may be different, and the LE spillage will occur before the trailing edge backflow. The following numerical results show that there is no trailing edge backflow at the last convergence point of the numerical results in this paper, but there is LE spillage, which may be caused by the steady calculation method. However, this paper does not pay attention to the stall process and unsteady fluctuation characteristics of the CRAC, and the verification of the numerical results and experimental results also show that the numerical method in this paper has high reliability. Therefore, in this paper, whether the LE spillage occurs or not is used to judge whether the compressor is under the unstable work condition or not, that is, whether the interface between the mainstream and the leakage flow is located in the blade channel or it spills from the LE. As shown in Hoying et al. (1999), the position of the large entropy gradient near the LE of the blade is used as the interface between the mainstream and TLV.

Fig. 9 presents the entropy contours and surface streamlines at $99 \%$ blade span of two rotors with different tip clearance sizes of rotor 1 , in which a red dashed line is used to denote the relative position of the interface between the leakage flow and the main flow, and the pink dashed circle marks the location where the LE spillage occurs. As can be seen from the Fig.9 (b), the loss at the blade tip 
increased in rotor 2 with the increase of blade tip clearance size of rotor 1 . The loss in rotor 2 mainly concentrates at the LE of the blade, which is due to the increase of tip clearance, the strength and influence range of TLV in rotor 1 become larger, resulting in the more complex inlet conditions of rotor 2 , thereby affecting the flow and loss around rotor $2 \mathrm{LE}$.

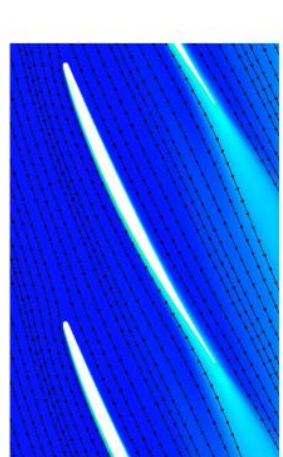

$0.25 \tau$

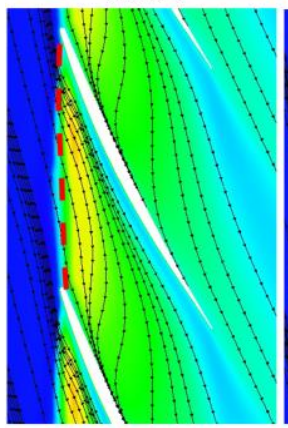

$1.75 \tau$

a) R1
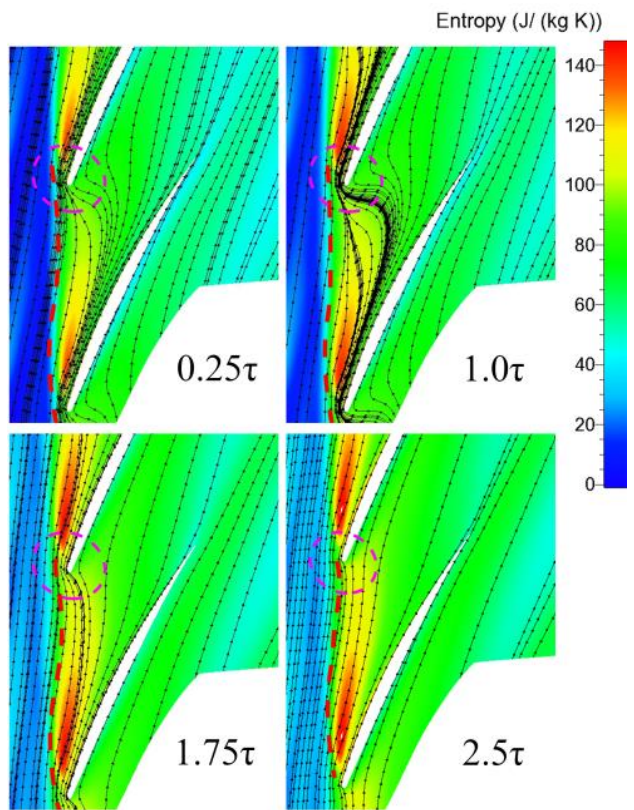

b) R2

Fig. 9. Surface streamlines and entropy contours on the blade tip near the stall condition with different tip clearance sizes in Rotor 1.
At the near stall point, when the tip clearance size of rotor 1 is $0.25 \tau, 1.0 \tau$, and $1.75 \tau$, there is no LE spillage at the tip of rotor 1 , and the interface between mainstream and leakage flow is still located in the blade channel. On the other hand, the interface of rotor 2 has been already pushed out of the blade LE plane and the LE spillage phenomenon happened. which indicates that the rotor 2 reaches the stall condition first under these three kinds of tip clearance size, that is, the first stall stage of the rotor compressor is rotor 2. However, when the tip clearance size of rotor 1 is $2.5 \tau$, the LE spillage phenomenon appears at the tip of rotor 1 , and the interface is basically parallel to the inlet of the blade, and completely located outside the blade channel. Meanwhile, rotor 2 also has the phenomenon of LE spillage. Hoying et al. (1999) found that the stall precursor is related to the stability of the TLV, and the development of the TLV in the blade channel is restricted by the axial velocity of the main flow. There is a balance between the axial velocity component of tip leakage vortex and the main flow. When the compressor outlet back pressure increases, the ability of leakage flow to expand and develop in the blade channel is enhanced. On the other hand, as the mainstream flow into the blade channel decreases, the axial component of the mainstream velocity is less able to suppress disturbance. As a result, the original balance is broken, and the interface between the leakage flow and the mainstream gradually moves towards the LE of the blade until it completely leaves the blade channel, and then the spike-type stall is induced.

By comparing the interface at the LE of rotor 1 and rotor 2 , it can be found that the interface of rotor 1 is farther away from the inlet and completely parallel to the inlet, and there exists an obvious LE spillage phenomenon. On the other hand, the interface of rotor 2 is still concave into the blade channel due to mainstream suppression, which indicates that the axial component of mainstream velocity at the tip of rotor 2 still has a strong ability to suppress disturbance, and no obvious LE spillage has been formed. Therefore, it can be considered that the first stall stage is rotor 1 when the tip clearance is $2.5 \tau$.

The expansion and fragmentation of TLF and the accumulation of low energy fluid at the blade tip are important factors leading to the blockage of the channel at the tip of the blade. In order to analyze the reasons for the blockage of the flow passage and the induction of the first stall stage, Fig. 10 and Fig. 11 shows the absolute vorticity distribution on cross flow planes of the rotor channel and the streamline trajectory diagram released from the LE in the middle of the tip clearance with rotor 1 tip clearance size of $1.0 \tau$ and $2.5 \tau$ respectively, where the streamline color represents the value of the standard helicity. Absolute vorticity $\Omega_{n}$ and standard helicity $\xi_{n}$ are defined as follows

$\Omega_{n}=\frac{|\vec{\xi}|}{2 \omega}$ 


$$
\xi_{n}=\left|\frac{\vec{\xi} \cdot \vec{w}}{|\vec{\xi}||\vec{w}|}\right|
$$

Where $\vec{\xi}$ and $\vec{w}$ represents the absolute vorticity vector and the relative velocity vector respectively, and $\omega$ represents the angular velocity of the rotor.

The high absolute vorticity region corresponds to the tip leakage vortex core, and the larger the absolute vorticity value is, the higher the tip leakage vorticity intensity is. The direction of absolute vorticity vector represents the direction of the leakage vortices determined by the right-handed helix rule, and the relative velocity vector represents the direction of the local velocity of the airflow in the cylindrical coordinate system. Since the secondary flow in the rotor is mainly controlled by the component of absolute vorticity along the direction of the relative airflow, $\xi_{n}$ is defined and characterized by the two vectors of absolute vorticity and relative velocity. According to the definition formula, the standard helicity represents the cosine value of the angle between absolute vorticity vector and relative velocity, which means that the absolute value of the standard helicity of the fluid moving along the vorticity line is 1 while moving perpendicular is 0 .
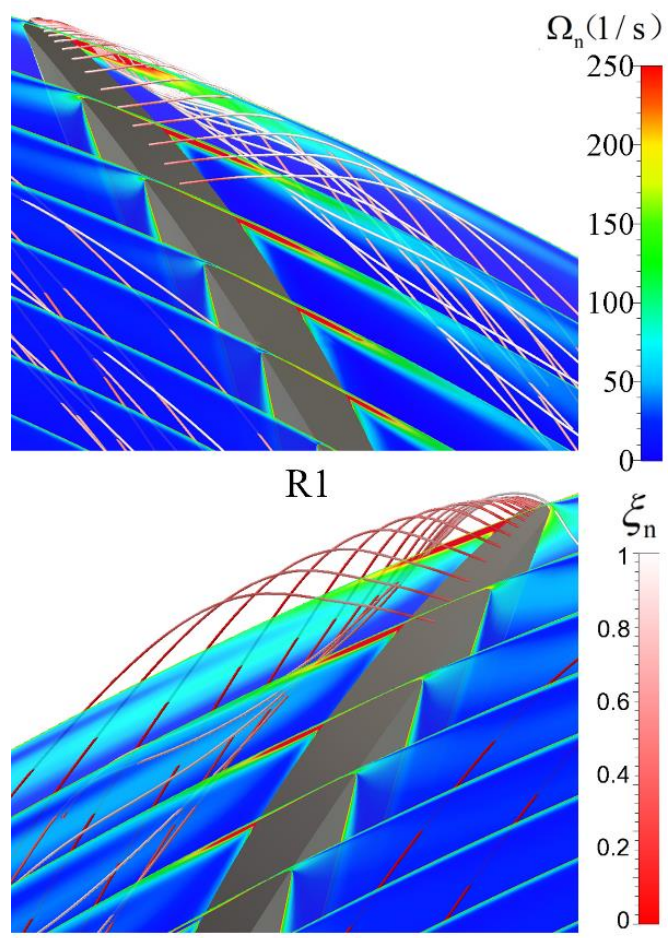

$\mathrm{R} 2$

Fig. 10. Absolute vorticity distribution on cross flow planes and variations of normalized helicity along the tip leakage streamlines with rotor 1 tip clearance size of $1.0 \tau$.
It can be seen from Fig.10 that under the near stall condition, the absolute vorticity in the rotor 1 channel gradually mixes, diffuses and weakens with the mainstream as it moves downstream along the flow direction when the rotor 1 is at the design clearance $\tau$. The value of $\xi_{n}$ on the streamline is always in the range of $0.8-1$, which shows that the TLV is always a concentrated vortex structure in the downstream propagation process. At this time, it can be found that the absolute vorticity in most regions near the LE of rotor 2 channel is obviously stronger than that at the LE of rotor 1 , and the region of high absolute vorticity is smaller and shrinks faster. The reason for this result is that the TLF intensity of rotor 2 is higher and the uniformity of inlet flow field is poor. In addition, because of the large gradient of absolute vorticity at the LE of rotor 2, the tip leakage vortex begins to expand from the LE of the blade. The corresponding standard helicity on the streamline is about 0.3 , indicating that the compactness of the streamline wound around the TLV vortex core begins to decrease from the LE to 0.2 near the $50 \%$ axial chord location. And the streamline will begin to disperse greatly and produce the secondary leakage phenomenon in the subsequent development, which leads to the blockage of the flow channel, but this phenomenon does not occur in rotor 1 . This is consistent with the result that rotor 2 is the first stall stage under this tip clearance in the previous analysis.

When the tip clearance size of rotor 1 is $2.5 \tau$, the flow structure in the blade channel of rotor 2 is basically the same as that of tip clearance size $1.0 \tau$, but the intensity of secondary leakage increases, which corresponds to the conclusion of a slight loss increase in Fig. 9 (b). At this time, the flow structure in the rotor 1 channel of the rotor changes greatly, and it can be seen from Fig.11 that the gradient of the absolute vorticity at the LE of the blade is larger, and the corresponding standard helicity on the clearance leakage streamline is about 0.15 , This means that the degree of the streamline winding around the TLV vortex core begins to decrease from the LE, and the streamline will start to expand and diverge, resulting in the phenomenon of secondary leakage or multiple leakage with the development of flow. On the other hand, the standard helicity in rotor 2 is about 0.82 , which is in the form of concentrated vortex. Compared with the flow in rotor 1 and rotor 2 , it is found that the rotor 1 is the most likely to reach the stall state first, which is consistent with the conclusion in Fig.9. Therefore, the change of the tip clearance of rotor 1 can change the first stall stage of CRAC.

\subsection{Effect of Rotor 2 Tip Clearance Change on CRAC}

Fig. 12 shows the static pressure coefficient contours of the blade tip near the peak efficiency point with varying tip clearance sizes for rotor 2 . It can be seen from Fig.12 (b) that when the tip clearance of rotor 2 is $0.25 \tau$, there is no obvious leakage flow trajectory in the passage of rotor 2 , 
and the initial position of TLV in rotor 2 moves backward with the increase of tip clearance. When the tip clearance increases to $2.5 \tau$, the starting position of TLV moves downstream obviously than that of $1 \tau$ and $1.75 \tau$, and the static pressure chute is steeper in this process, indicating that the intensity of TLF increase. We can also see that the trajectory of the leakage flow deflects along the circumferential to LE with the increase of the tip clearance, but the corresponding leakage flow trajectory deflects downstream when the tip clearance increases to $2.5 \tau$. This is different from the phenomenon that the TLV trajectory in the rotor 1 channel uniformly deflects upstream with the tip clearance of the rotor 1 increases. The reason for this result may be that rotor 2 is located at downstream and will be affected by the flow in upstream rotor 1. By observing Fig. 12 (a), it can be found that when the tip clearance of rotor 2 reaches $2.5 \tau$, the initial position of TLV in rotor 1 moves backward, with the strength is basically unchanged, which reduces the effect of TLV on the outlet of rotor 1 to some extent. Therefore, the inlet condition at the LE of rotor 2 is improved, so the TLV trajectory in rotor 2 is deflected downstream compared with other tip clearance sizes. In summary, it can be concluded that the flow in rotor 2 is more complex, which will be affected not only by the tip clearance size of itself, but also by the flow of rotor 1 in the upstream.
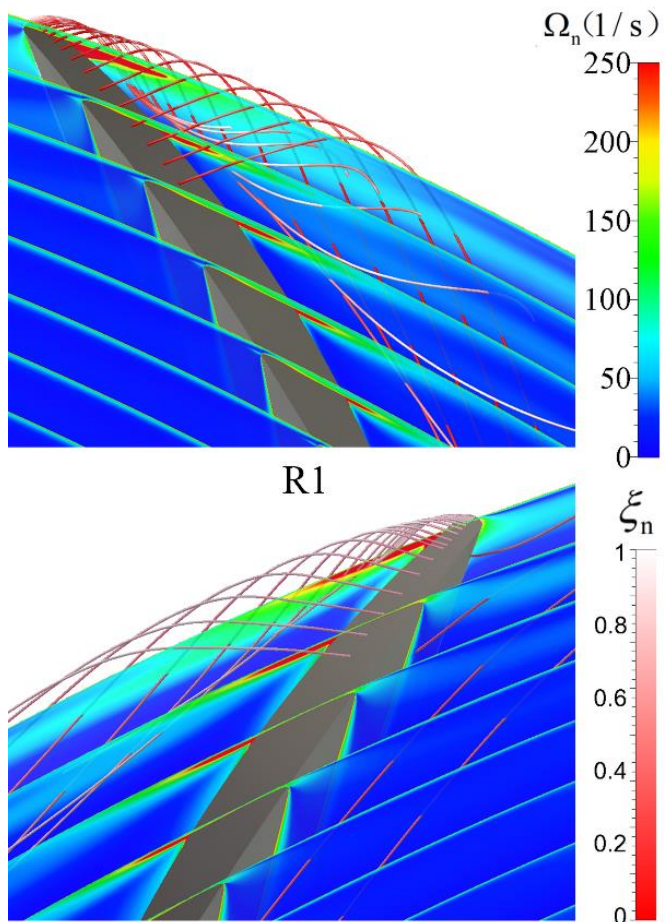

$\mathrm{R} 2$

Fig. 11. Absolute vorticity distribution on cross flow planes and variations of normalized helicity along the tip leakage streamlines with rotor 1 tip clearance size of $2.5 \tau$.

Figure 13 shows the TLVR along the chord length in the middle of the rotor 1 tip clearance when rotor 2 takes different tip clearance sizes at the peak efficiency point. It can be seen that when the tip clearance size of rotor 2 reaches $2.5 \tau$, the maximum leakage velocity position at the tip of rotor 1 moves backward, which is consistent with the conclusion that the starting position of TLV moves backward in Fig. 12 (a), and the leakage velocity away from the inlet increases significantly. Fig. 14 presents the static pressure coefficient at the blade tip of rotor 1 at the peak efficiency condition with different tip clearance sizes of rotor 2 . One can see that when the tip clearance of rotor 2 reaches $2.5 \tau$, the position of the maximum tip blade load moves backward significantly. In addition, the back pressure at the trailing edge of rotor 1 decreases, which reduces the inverse pressure gradient and enhances the flow capacity at the blade tip. All of these leads to the downstream shift of the TLV starting position in rotor 1 .

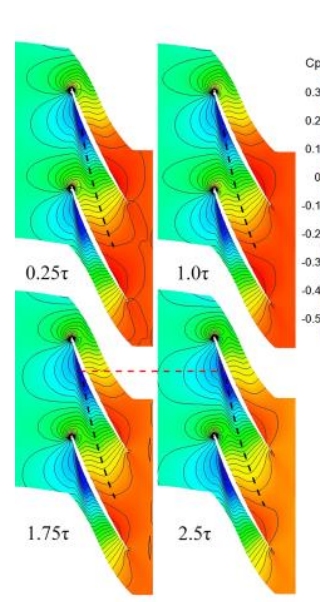

a) R1

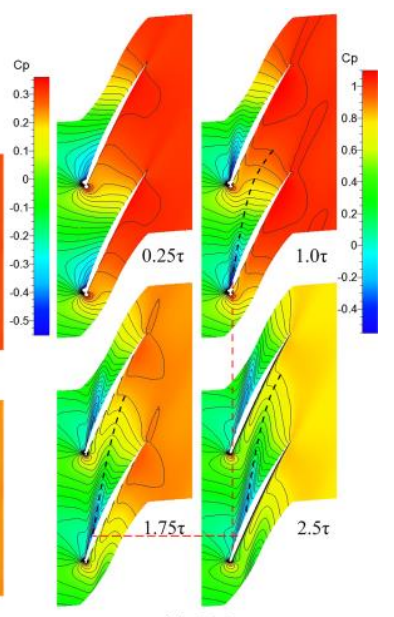

b) R2
Fig. 12. Static pressure coefficient contours of the blade tip near the peak efficiency point with different tip clearance sizes in Rotor 2.

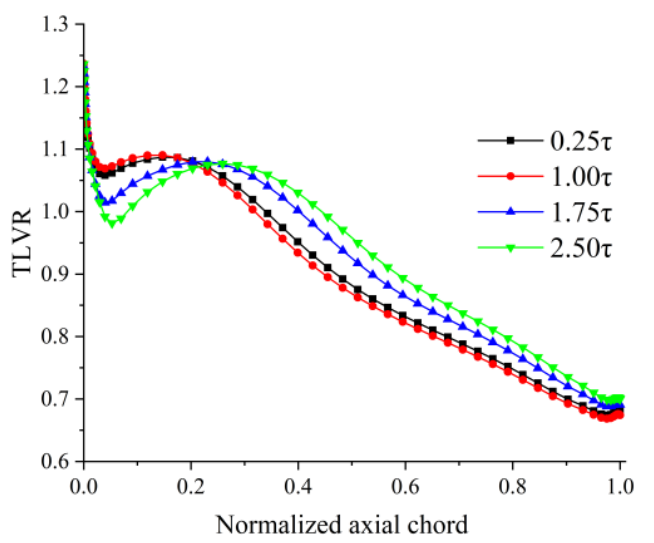

Fig. 13. TLVR distribution along with axial chord at the middle of the tip clearance sizes of rotor 1 at peak efficiency condition.

Figure 15 presents the entropy contours and surface streamlines at $99 \%$ blade span of two rotors with 
varying tip clearance sizes for rotor 2 . It can be seen that when the tip clearance of rotor 2 is $0.5 \tau$, the LE spillage phenomenon appears at the tip of rotor 1 , and the interface of rotor 1 has been separated from the blade channel and is basically parallel to the inlet. On the other hand, the interface of rotor 2 is still suppressed by the mainstream in the blade channel as shown in Fig. 15(b), which indicates that the axial component of mainstream velocity at the tip of rotor 2 still has the ability to suppress disturbance. While the axial component of mainstream velocity in rotor 1 is already perpendicular to the vortex line. The ability to suppress disturbance disappeared, therefore, it can be considered that the first stall stage is rotor 1 when the rotor 2 tip clearance size is $0.5 \tau$. When the rotor 2 tip clearance size increases to $1.0 \tau, 1.75 \tau$ and $2.5 \tau$, the LE of the rotor 1 doesn't exist spillage, but all the interface at the tip of the rotor 2 has been pushed out from the inlet, indicating that the rotor 2 is the first to reach the stall condition under these three kinds of tip clearance sizes. That is, the first stall stage of the CRAC is rotor 2.

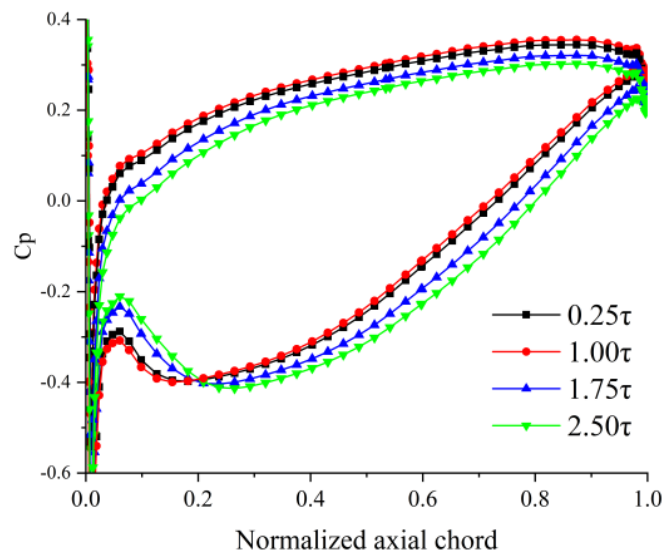

Fig. 14. Static pressure coefficient at the blade tip of rotor 1 at the peak efficiency condition with different tip clearance sizes in rotor 2 .

Fig. 16 shows the absolute vorticity distribution on crossflow planes of the rotor channel and variations of normalized helicity along the tip leakage streamlines released from the LE in the middle of the tip clearance with rotor 2 tip clearance size at $0.25 \tau$. One can that under the near stall condition, when the tip clearance of rotor 2 is $0.25 \tau$, the absolute vorticity in the rotor 2 channel of the rotor gradually mixes and weakens with the mainstream as it moves downstream along the flow direction. The standard helix value on the streamline decreases gradually from 0.95 . It can be seen that there is no expansion and fragmentation in the downstream propagation of TLV. However, in rotor 1 , with the development of TLV downstream, the absolute vorticity decreases gradually, and the corresponding standard helicity at the LE streamline is about 0.2. It means that the streamline that develops near the middle and rear chord length will gradually begin to expand, disperse and produce secondary leakage, which will lead to the blockage and stall of the flow channel. This is consistent with the result that rotor 1 is the first stall stage under the tip clearance $0.25 \tau$ in the previous analysis. Figure 10 has presented the variations of normalized helicity along the tip leakage streamlines with both rotor 1 and rotor 2 tip clearances are design gap $1.0 \tau$, as analyzed earlier. At this tip clearance size, the standard helix value on the streamline of rotor 1 is always kept at 0.95 to 1 . It can be considered that there is no expansion and breakage in the downstream propagation of TLV, and it is always maintained as a concentrated vortex structure. Therefore, rotor 2 is the first stall stage under the tip clearance size $1.0 \tau$, which is consistent with the conclusion in Fig. 15. So, the change of rotor 2 tip clearance can also change the first stall stage.

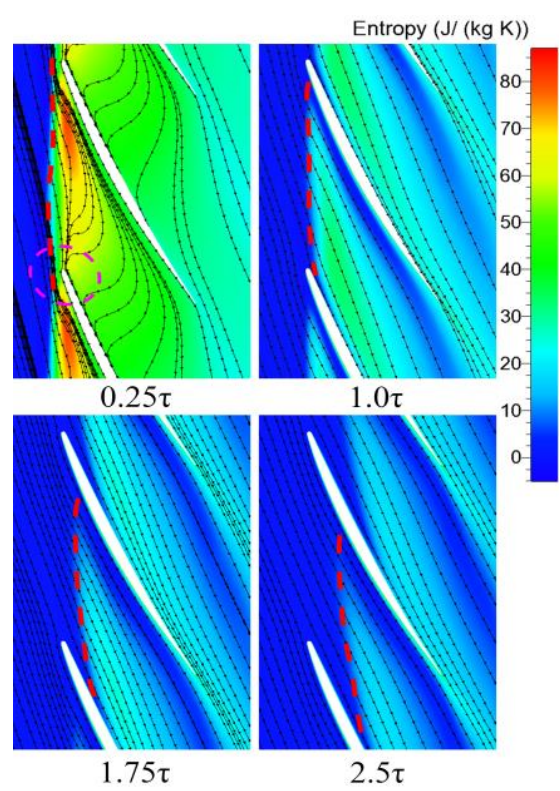

a) R1

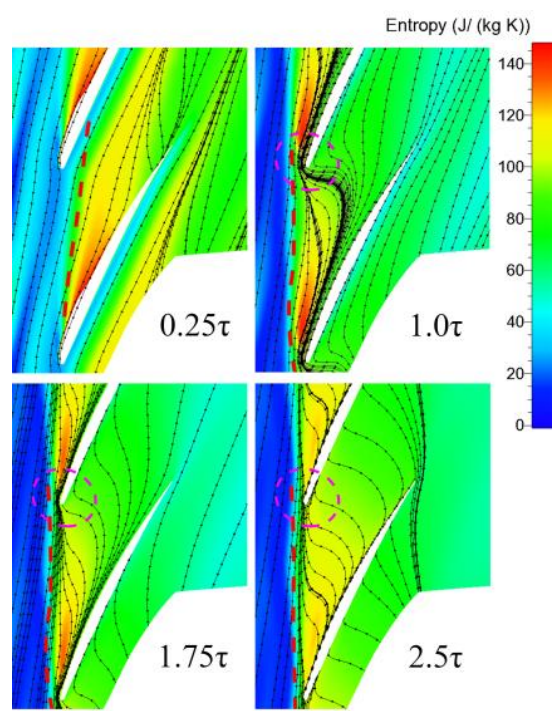

b) R2

Fig. 15. Surface streamlines and entropy contours on the blade tip near the stall condition with different tip clearance sizes in rotor 2. 

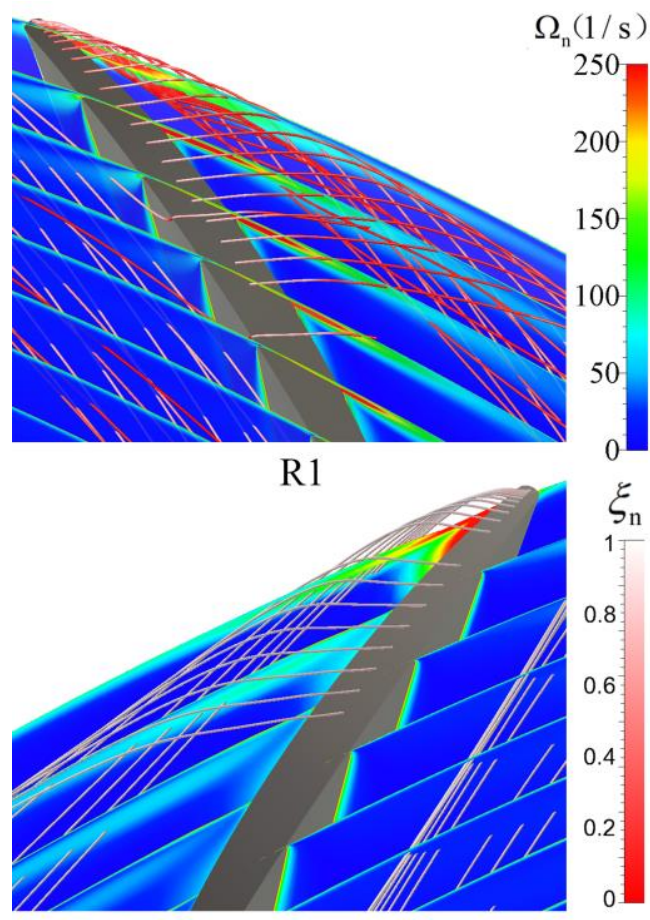

$\mathrm{R} 2$

Fig. 16. Absolute vorticity distribution on crossflow planes and variations of normalized helicity along the tip leakage streamlines with rotor 2 tip clearance size at $0.25 \tau$.

\section{CONClusion}

In this paper, a numerical investigation has been done to explore the effects of the tip clearance variation of different rotors on the performance of a counter-rotating axial compressor. Both the compressor total pressure ratio and efficiency decrease with the increase of tip clearance, and the peak efficiency sensitivity curve of the two rotors is approximately negative linear with the tip clearance size, and the slope of the peak efficiency and surge margin sensitivity curve corresponding to rotor 2 is obviously larger than that of the rotor 1 . There exists an optimum tip gap combination for this counter-rotating axial compressor, i.e. $0.5 \tau$ for Rotor 1 and $0.25 \tau$ for rotor 2, and the peak efficiency and stability margin are improved by about $0.63 \%$ and $29.4 \%$, respectively. The increase of tip clearance size will make the initial position of the TLV of that rotor shifted downstream with the TLV scale and intensity become larger, and the TLV trajectory will deflect to inlet along with the circumferential. For one of the rotors in this counter-rotating axial compressor, the tip flow is influenced by the tip clearance variation of the other rotor. In addition, the effect is more obvious at the near stall point than at the peak efficiency point. The first rotating stall stage is also influenced by the tip clearance variation of both rotor 1 and rotor 2 . When the rotor 1 tip clearance is large or the rotor 2 tip clearance is small, the first stall stage will change from rotor 2 to rotor 1 .

The main purpose of the present paper is to explore the sensitivity to the tip clearance size change of each individual rotor and the corresponding aerodynamic mechanisms associated with the performance variation in the CRAC. The validation of the numerical approach with experimental results gives us the confidence to study the effects of tip clearance size, and the above conclusions may provide guidance for the design in CRAC. It should be mentioned that the effect of modifying both rotors tip clearance size was not considered for the brevity of the paper. Therefore, to better understand the effects of tip clearance size on the performance and stability enhancement of CRAC, both the detailed influence of the simultaneous tip clearance size changes of two rotors and the unsteady effects will be studied in the future with numerical and experimental methods.

\section{ACKNOWLEDGEMENTS}

This paper is supported by the National Natural Foundation of China (Project No: 51676162, No.51790512) and Collaborative Innovation Center of Advanced Aero-Engine in Beijing, these supports are gratefully acknowledged.

\section{REFERENCES}

Anson, M. and L. Zhang (1995). On-site graphics for planning and communicating the use of site space. In Y. Loo (Ed.), Proceedings of the Fifth East Asia-Pacific Conference on Structural Engineering and Construction, Gold Coast, Australia, 883-888. Griffith University.

Berdanier, R. and N. Key (2016). The effects of tip leakage flow on the performance of multistage compressors used in small core engine applications. Journal of Engineering for Gas Turbines and Power 138, 052605.

Boudet, J., A. Cahuzac, P. Kausche and C. Marc (2015). Zonal large-eddy simulation of a fan tip-clearance flow with evidence of vortex wandering. Journal of Turbomachinery 137: 061001 .

Brandt, H., L. Fottner, H. Saathoff and U. Stark (2002). Effects of the inlet flow conditions on the tip clearance flow of an isolated compressor rotor. ASME Paper No. GT200230639.

Camp, T. and I. Day (1997). A study of spike and modal stall phenomena in a low- speed axial compressor. Journal of Turbomachinery 120 (3):393-401.

Deng, X., L. Gao, L. Gao and Y. Cai (2013). Numerical investigation of parameters transmission on blade rows interface of contrarotating compressor. Journal of aerospace power 30(6), 1460-1469.

Domercq, O. and J. F. Escuret (2007) Tip clearance effect on high-pressure compressor stage matching, Proceedings of the Institution of 
T. Liang et al. / JAFM, Vol. 13, No. 5, pp. 1587-1599, 2020.

Mechanical Engineers, Part A: Journal of Power and Energy 221(6), pp. 759-767.

Du, H., X. Yu, Z. Zhang and B. Liu (2013). Relationship between the flow blockage of tip leakage vortex and its evolutionary procedures inside the rotor passage of a subsonic axial compressor. Journal of Thermal Science 22: $522-531$.

Gao, L., X. Li, X. Feng and B. Liu (2012). The effect of tip clearance on the performance of contra-rotating compressor. ASME Paper No. GT2012-68801.

Hoying, D., C. Tan, H. Vo and E. Greitzer (1999). Role of blade passage flow structurs in axial compressor rotating stall inception. Journal of Turbomachinery 121(4), 735-742.

Inoue, M. and M. Kuroumaru (1989). Structure of tip clearance flow in an isolated axial compressor rotor. Journal of Turbomachinery 111(3): 250-256.

Joly, M., T. Verstraete and G. Paniagua (2013). Full design of a highly loaded and compact contrarotating fan using multidisciplinary evolutionary optimization. ASME Paper No. GT2013-94433.

Lejon, M., N. Andersson, L. Eriksson and L. Ellbrant (2015). Simulation of tip-clearance effects in a transonic compressor. ASME Paper No. GT2015-43033.

Mistry, C. and A. Pradeep (2014). Influence of circumferential inflow distortion on the performance of a low speed, high aspect ratio contra rotating axial fan. Journal of Turbomachinery 136, 071009.

Nouri, H., A. Danlos, F. Ravelet, F. Bakir and C. Sarraf (2013). Experimental study of the instationary flow between two ducted counterrotating rotors. Journal of Engineering for Gas Turbines and Power 135, 022601.

Pundhir, D. and P. Sharma (2013). A study of aerodynamic performance of a contra rotating axial compressor stage. Defence Science Journal 42 (3): 191-199.
Sakulkaew, S., C. Tan, E. Donahoo, C. Cornelius, and M. Montgomery (2013). Compressor efficiency variation with rotor tip gap from vanishing to large clearance. Journal of Turbomachinery 135: 031030.

Schimming, P. (2003). Counter rotating fans-an aircraft propulsion for the future? Journal of Thermal Science 12, 97-103.

Sharma, P., Y. Jain and D. Pundhir (1988). A study of some factors affecting the performance of a contra-rotating axial compressor stage. Proceedings of the Institution of Mechanical Engineers, Part A: Power and Process Engineering 202(1), 15-21.

Shi, L., B. Liu, Z. Na, X. Wu and X. Lu (2015). Experimental investigation of a counterrotating compressor with boundary layer suction. Chinese Journal of Aeronautics 28, 1044-1054.

Storer, J. and N. Cumpsty (1994). An approximate analysis and prediction method for tip clearance loss in axial compressors. Journal of Turbomachinery 116(4), 648-656.

Tan, D., Y. Li, I. Wilkes, R. Miorini and J. Katz (2015). Visualization and time resolved particle image velocimetry measurements of the flow in the tip region of a subsonic compressor rotor. Journal of Turbomachinery 137, 041007.

Vo, H., C. Tan and E. Greitzer (2008). Criteria for spike initiated rotating stall. Journal of Turbomachinery 130: 011023.

Wang, Y., W. Chen, C. Wu and S. Ren (2015). Effects of tip clearance size on the performance and tip leakage vortex in dualrows counter-rotating compressor. Proceedings of the Institution of Mechanical Engineers, Part G: Journal of Aerospace Engineering 229(11), 1953-1965.

Yamada, K., H. Kikuta, M. Furukawa, S. Gunjishima and Y. Hara (2013). Effects of tip clearance on the stall inception process in an axial compressor rotor. ASME Paper No. GT2013-95479. 